\title{
Unveiling universal trends for the energy level alignment in organic/oxide interfaces
}

\author{
José I. Martínez, ${ }^{\mathrm{a},{ }^{*}}$ Fernando Flores, ${ }^{\mathrm{b},{ }^{+}}$José Ortega, ${ }^{\mathrm{b}}$ Sylvie Rangan, ${ }^{\mathrm{c}}$ \\ Charles M. Ruggieri, ${ }^{c}$ and Robert A. Bartynski ${ }^{c}$ \\ ${ }^{a}$ Materials Science Factory, Dept. Surfaces, Coatings and Molecular Astrophysics, \\ Institute of Material Science of Madrid (ICMM-CSIC), Sor Juana Inés de la Cruz 3, \\ 28049 Madrid (Spain) \\ ${ }^{b}$ Dept. Física Teórica de la Materia Condensada and Condensed Matter Physics Center \\ (IFIMAC), Universidad Autónoma de Madrid, 28049 Madrid (Spain) \\ ${ }^{c}$ Dept. Physics and Astronomy, and Laboratory for Surface Modification, Rutgers, The \\ State University of New Jersey, Piscataway, NJ 08854-8019 (USA) \\ *E-mail: joseignacio.martinez@icmm.csic.es \\ † E-mail: fernando.flores@uam.es
}

In this perspective we present a comprehensive analysis of the energy level alignment at the interface between an organic monolayer (organic = perylenetetracarboxylic dianhydride, PTCDA, zinc tetraphenylporphyrin, Zn-TPP, and tetracyanoquinodimethane, TCNQ) and a prototypical oxide surface, $\mathrm{TiO}_{2}(110)$, looking for universal behaviours. PTCDA shows a physisorbed interaction with $\mathrm{TiO}_{2}$ and a small interface dipole potential with its highest occupied molecular orbital (HOMO) energy level located in the oxide energy gap and the lowest occupied molecular orbital (LUMO) energy level located above the oxide conduction band minimum, $E_{C}$. We analyse how the interface barrier depends on an external bias potential between the organic layer and the oxide surface, $\Delta$, and find for this interface that the screening parameter $S=d /\left(E_{C}-H O M O\right) / d \Delta$ is close to 1 . In the second case, the Zn-TPP monolayer shows a moderate chemisorbed interaction with some charge transfer from the molecule to the oxide and a significant interface dipole potential, in such a way that $S$ decreases to around 0.8 . In the TCNO/TiO $2(110)$ case, the TCNQ molecules present a strong chemical interaction with the oxide; the LUMO energy level is located in the oxide energy gap in such a way that one electron is transferred from the oxide to the organic molecule; we also find that in this case $S \approx 0.5$. All these cases can be integrated within a universal behaviour when $\left(E_{C}-H O M O\right)$ is calculated as a function of $\Delta$; that function presents a zig-zag curve with a central part having an $S$-slope, and two plateaus associated with either the LUMO or the HOMO energy levels crossing the oxide Fermi level. In these plateaus, a Coulomb blockade regime arises and a space charge layer develops in the oxide surface.

\section{Introduction}

Due to their extended $\pi$-systems, polyaromatic molecules appear as convenient organic semiconductor materials for many technological applications such as organic electronics and optoelectronics. ${ }^{1-5}$ Recently, hybrid organic-inorganic materials consisting of the combination of organic semiconductors and transition metal oxides 
have attracted great interest due to their complementary properties. Organic semiconductors present a large variety in their properties and a low cost and ease of production. Many organic materials interact strongly with light, a property of special interest for light-emitting and light-harvesting devices. Transition metal oxides have a great potential for organic electronics due to their good and tuneable capabilities for charge exchange with organic semiconductors, their semiconducting properties and good transparency. ${ }^{4}$

Although the intrinsic bulk properties of the organic material, such as the molecular packing and electronic structure, determine energy adsorption and carrier propagation mechanisms, a critical factor determining the properties of hybrid organic/inorganic systems is the relative positioning of the energy levels corresponding to the frontier states at the interface between both materials. ${ }^{3-5}$ There has been a lot of effort to investigate energy level alignment in organic/metal and organic/organic interfaces, ${ }^{6-8}$ and the results have been explained using models such as the Induced Density of Interface States (IDIS) ${ }^{7}$ or Integer Charge Transfer (ICT). ${ }^{9}$ A major contribution shifting the organic energy levels is the dipole potential due to charge transfer at the interface, ${ }^{7}$ the molecular dipoles or multipoles, ${ }^{10}$ and the distortion of the metal surface charge due to the Pauli repulsion ("pillow" effect) ${ }^{11}$ may also yield a relevant contribution to this interface potential. The polarization response of the system, shifting occupied and empty states in opposite directions, ${ }^{7}$ and the hybridization of the molecule orbitals at the interface, ${ }^{3}$ also play an important role in energy level alignment.

In contrast, the mechanisms of energy level alignment for organic/oxide interfaces still remain poorly understood and are still challenging, and have been under study in recent years . . $^{32-13}$ This case can be expected to present important similarities to the organic/metal and organic/organic interfaces, but also some differences. For example, it has been suggested that the organic electronegativity and the electron chemical potential equilibration between the oxide Fermi level and organic ionization energy play a preeminent role for energy level alignment for organic/oxide interfaces. ${ }^{12}$ However, effects such as electron charge transfer upon molecular adsorption with possible space charge layer formation in the oxide, as well as the nature of the chemical interaction of the organic on the oxide surface, are important considerations in any attempt to fully understand such interfaces. ${ }^{13-18}$

In this perspective we analyse the physicochemical mechanisms that control energy level alignment at the interface between a prototypical metal oxide surface, $\mathrm{TiO}_{2}(110)$, and different organic monolayers with dissimilar properties, PTCDA, ${ }^{18} \mathrm{Zn}-\mathrm{TPP}^{15}$ and TCNQ. ${ }^{14}$ These organic materials present very different electronegativity values and can be considered as paradigmatic and representative examples for the broad diversity of electronic properties that organic/oxide interfaces present and for the analysis of the organic/oxide behaviour. Moreover, the chemical interaction of these organic layers with the oxide surface ranges from physisorption (PTCDA) to strong chemical interaction (TCNQ), with Zn-TPP presenting a moderate intermediate chemical interaction. These interfaces have been studied using a combination of experimental and theoretical techniques in previous publications. ${ }^{14-15,18}$ Herein, we review the main results and discuss the similarities and differences found in these different paradigmatic cases, putting these results in a more general perspective. Also, in order to get new insights (and better understand the connection between these different 
cases), we present a new proof-of-concept analysis in which we apply a "theoretical" bias potential between the organic and the oxide, $\Delta$, and analyse the energy level alignment at these interfaces as a function of $\Delta .{ }^{19}$ The discussion presented herein allows us to infer general properties and characterize a universal behaviour for organic/oxide interfaces.

\section{Experiments and Techniques}

The energy level alignment at the organic/ $/ \mathrm{TiO}_{2}$ interface was determined by means of ultraviolet photoemission spectroscopy and inverse photoemission spectroscopy measurements performed in a single UHV experimental system described elsewhere. ${ }^{14,15,47}$ In UPS and XPS, the kinetic energy of electrons ejected from the sample by excitation from monoenergetic photons reflects the density of occupied states in the system. In IPS the photon energy distribution associated with the decay of a monoenergetic beam of electrons directed to the sample reflects the density of unoccupied states.

Core levels were probed using X-ray photoemission spectroscopy excited by $\mathrm{Al} \mathrm{K \alpha}$ radiation, and the valence band electrons were examined using $\mathrm{He} I \mathrm{II}(40.8 \mathrm{eV})$ excited ultraviolet photoemission spectroscopy. ${ }^{14,15}$ In both cases, electron energy distribution was measured using a cylindrical mirror analyser. IPS was performed using a grating spectrometer with a primary electron energy of $20.3 \mathrm{eV}$. The overall energy resolution for the UPS and the IPS spectra is estimated to be better than 0.3 and $0.6 \mathrm{eV}$, respectively.

For both clean and sensitized surfaces, the secondary electron cut-off was measured on biased samples using $\mathrm{He} \mathrm{I}(21.1 \mathrm{eV})$ to determine the organic electron affinity and the induced interface dipole. ${ }^{14,15}$ In order to study the adsorbate geometry for some of the organic molecule, STM measurements were performed on an Omicron VT-STM at $300 \mathrm{~K}$. In some of the figures presented in this paper, we show the energy level alignment obtained from these experiments.

\section{Theoretical Approach and Models}

\section{Computational details}

For all the calculations of this study, devoted to the structural optimization and the computation of the electronic structure (including the correction of molecular gaps and adjusting the correct energy level alignment between substrate and adsorbates) of the different organic/ $\mathrm{TiO}_{2}$ interfaces discussed in this paper, Density Functional Theory (DFT) was used effectively combining the plane-wave and localized-basis-set schemes as implemented in the plane-wave QUANTUM ESPRESSO simulation package ${ }^{20}-$ used for the determination of the complex interface atomic geometries -, and in the localized-basis-set FIREBALL simulation code ${ }^{21-23}$ - employed to analyse the interface electronic properties after introducing appropriate correlation effects on the electronic levels of the organic molecule (see below).

For the plane-wave code QUANTUM ESPRESSO, ${ }^{20}$ one-electron wave-functions were expanded in a basis of plane-waves, with energy cut-offs of 400 and $500 \mathrm{eV}$ for the kinetic energy and for the electronic density, respectively, which have been adjusted 
to achieve sufficient accuracy in the total energy. The exchange-correlation (XC) effects have been accounted for by using the revised version of the generalized gradient corrected approximation (GGA) of Perdew-Burke-Ernzerhof (rPBE), ${ }^{24}$ and Rabe-RappeKaxiras-Joannopoulos (RRKJ) ultrasoft pseudopotentials ${ }^{25,26}$ have been considered to model the ion-electron interaction. In all the calculations, the Brillouin zones (BZ) were sampled by means of optimal Monkhorst-Pack grids ${ }^{27}$ guaranteeing a full convergence in energy and electronic density. A perturbative van der Waals (vdW) correction was used to check the reliability of all the adsorbed adlayer configurations. For this purpose, we have used an empirical efficient $\mathrm{vdW} \mathrm{R}^{-6}$ correction to add dispersive forces to conventional density functional (DFT+D). ${ }^{28,}{ }^{29}$ The atomic relaxations were carried out within a conjugate gradient minimization scheme, until the maximum force on any atom was below $0.01 \mathrm{eV} \AA^{-1}$. The Fermi level was smeared using the Methfessel-Paxton approach ${ }^{30}$ with a Gaussian width of $0.01 \mathrm{eV}$, and all energies were extrapolated to $\mathrm{T}=0 \mathrm{~K}$. Self-consistency in the electron density was converged up to a precision in the total energy better than $10^{-6} \mathrm{eV}$.

On the other hand, the computationally efficient FIREBALL code ${ }^{21-23}$ is based on a localorbital formulation in which self-consistency is implemented on the orbital occupation numbers. The main advantage of using this local-orbital code for our analysis of the different organic/ $/ \mathrm{TiO}_{2}(110)$ interfaces is that it offers an improved description of the electronic structure of organic semiconductor interfaces (in particular, the organic energy gap ${ }^{7}$ ) due to the inclusion in the code of appropriate corrections, see equations 1 and 2, for the electronic structure calculation. For these local-orbital calculations we have used a basis set of optimized numerical atomic orbitals (NAOs); ${ }^{31}$ in particular, $s p^{3} d^{5}$ NAOs for $\mathrm{C}, \mathrm{N}$ and $\mathrm{Ti}, s p^{3} s^{*} p^{3 *}$ NAOs for $\mathrm{O}$, and an optimized $s \mathrm{NAO}$ for $\mathrm{H}$ (see further details in previous recent literature ${ }^{14,15}$ ). Within the FIREBALL approach we have used the Local Density Approximation (LDA) functional ${ }^{21,32}$ and the ion-electron interaction has been modelled by means of norm-conserving scalar-relativistic pseudopotentials. ${ }^{33}$ We have used the quantum-chemistry all-electron cc-pVTZ/B3LYP model implemented in GAUSSIANO9 ${ }^{34}$ to obtain the HOMO (as the molecular ionization potential, $I P_{\text {molecule }}$ ) and the $L U M O$ (as the molecular electron affinity, $E A_{\text {molecule }}$ ) values for the gas-phase TCNQ, PTCDA and Zn-TPP molecules shown in Figure $1 \mathrm{a}$.

The $\mathrm{TiO}_{2}(110)-1 \times 1$ surface was modelled in a repeated slab geometry with: (i) a slab of five physical $\mathrm{TiO}_{2}(110)$ layers with a $25 \AA$ of vacuum between neighbouring cells along the axis perpendicular to the surface, as well as (ii) full periodic boundary conditions representing infinite $\mathrm{TiO}_{2}(110)$ surfaces. In all cases, a perfectly $\mathrm{TiO}_{2}$ balanced stoichiometry was used in order to avoid surface polarization effects. For the full geometry optimizations only the three bottom $\mathrm{TiO}_{2}$ physical layers were kept fixed in the calculations, in such a way that all the non-fixed atoms were free to move up to achieve residual forces lower than $0.01 \mathrm{eV} / \AA$. Additionally, in order to check the total energy results and electronic structure for the optimized geometrical configurations, ground-state calculations were recalculated by including an additional oxide layer in all the cases, with no significant variations with respect to the five physical substrate layers case, guaranteeing the energy convergence in the results for the different configurations (with an estimated uncertainty below $0.01 \mathrm{eV}$ ).

\section{Molecular gap correction and energy level alignment approach}


Once the optimal ground-state organic/ $/ \mathrm{TiO}_{2}$ geometries have been established, the organic/oxide interface electronic properties are analysed introducing some corrections within our local-orbital DFT calculations. These corrections are applied to overcome the following well-known limitations of conventional DFT implementations (e.g. LDA, GGA): (a) the Kohn-Sham energy levels yield transport gaps for the organic molecules that are usually too small; ${ }^{7,35,36}$ and (b) the initial relative level alignment between the oxide and the organic materials is poorly described even in well converged LDA (or GGA) calculations. ${ }^{37-40}$ For this purpose, in recent publications by our group (see e.g. Refs. ${ }^{14,15,35,36,41}$ ), we have introduced the two following operators to address the mentioned problems. With the following scissor operator:

$$
O^{\text {scissor }}=\sum_{(\mu v)}\left\{\left(\frac{U}{2}\right)|\mu\rangle\left\langle\mu\left|-\left(\frac{U}{2}\right)\right| v\right\rangle\langle v|\right\},
$$

with () being the empty (occupied) molecular orbitals of the isolated molecule (with the actual geometry of the molecule on the surface), we open the LDA-energy gap, $E_{g}{ }^{L D A}$, to $E_{g}{ }^{L D A}+U$, where $U$ is fixed to yield the experimental energy gap of the organic material adsorbed on the oxide (see below). We should mention that this energy gap is smaller than the one found for an isolated molecule, because in this case the effective $U$ is not screened by the interface dielectric response (see Figure 1). Additionally, the following shift operator:

$$
O^{\text {shift }}=\sum_{(\mu \nu)}\{(\varepsilon)(|\mu\rangle\langle\mu|+| v\rangle\langle v|)\}
$$

introduces a rigid shift, $\varepsilon$, of the molecular levels; $\varepsilon$ is fixed to yield the appropriate initial alignment, as provided by the experimental evidence, between the oxide and the organic energy levels of the deformed molecule as obtained in our calculations; ${ }^{14}$, 15,18 that initial alignment corresponds to an ideal situation for which the organic/oxide interaction has not yet been switched on. These corrections are introduced in the local-orbital Hamiltonian, and the interface electronic structure is then obtained by means of a self-consistent DFT calculation using the interface atomic geometry obtained with the QUANTUM ESPRESSO code. ${ }^{20}$ It should be stressed that in our theoretical calculations the oxide energy gap is $3.0 \mathrm{eV}$, while in the experiments a gap of $3.6 \mathrm{eV}$ is observed; this discrepancy is taken into account in our calculations by taking as a reference level for the oxide, in the initial oxide/organic alignment, the oxide middle of the gap except for $\mathrm{TCNQ} / \mathrm{TiO}_{2}$, where, due to its particular alignment (see below), the oxide conduction band edge is used as the reference level. 

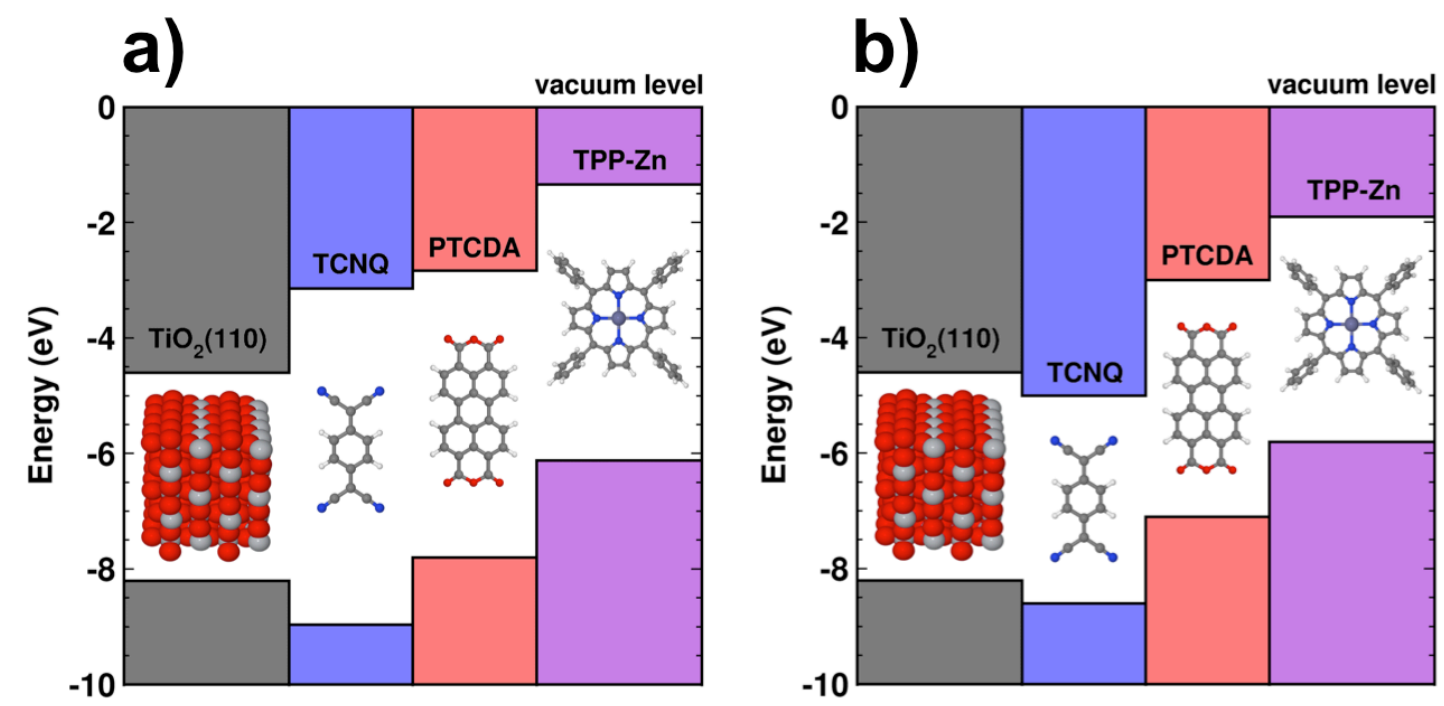

Figure 1. Valence and conduction bands for the $\mathrm{TiO}_{2}(110)$ surface and HOMO and LUMO levels of the organic materials (TCNQ, PTCDA and Zn-TPP) for (a) their gas phase and (b) the organic/TiO ${ }_{2}(110)$ interface. These interface values are taken from the experimental evidence. The organic energy gap for the interface case is smaller than the one for the isolated molecule due to the interface screening that reduces the value of $U\left(E_{g}=E_{g}{ }^{L D A}+U\right)$.

\section{Results for Different Organic Molecules (PTCDA, Zn-TPP and TCNQ)}

\section{PTCDA/TiO 2 (110) interface (physisorbed regime)}

Recent works ${ }^{42,} 43$ have proposed two likely candidates for PTCDA adsorption on $\mathrm{TiO}_{2}(110)$. In one case, PTCDA molecules are adsorbed on the oxide surface lying along one of their long sides in a tilted geometry, see Figure 2, in similarity to the cases of PTCDI, perylene or pentacene. ${ }^{44-46}$ In the second case, PTCDA molecules present a flat lying geometry on the surface. ${ }^{43}$ Our calculations ${ }^{18}$ for the two cases show different properties: while the tilted geometry has a weak interaction with the surface, the flat geometry develops a stronger one, with a significant molecular distortion. The interface energy level alignment is very sensitive to the adsorption geometry: the flat geometry presents a much larger interface dipole potential $(1.7 \mathrm{eV})$ than the tilted case $(0.3 \mathrm{eV})$, due to a larger charge transfer between the molecule and the oxide. Comparison with the experimental evidence ${ }^{18}$ (see Figure 3 ) suggests that the physisorbed adsorption (tilted geometry) is the most likely candidate for the PTCDA adsorption on $\mathrm{TiO}_{2}(110)$. 

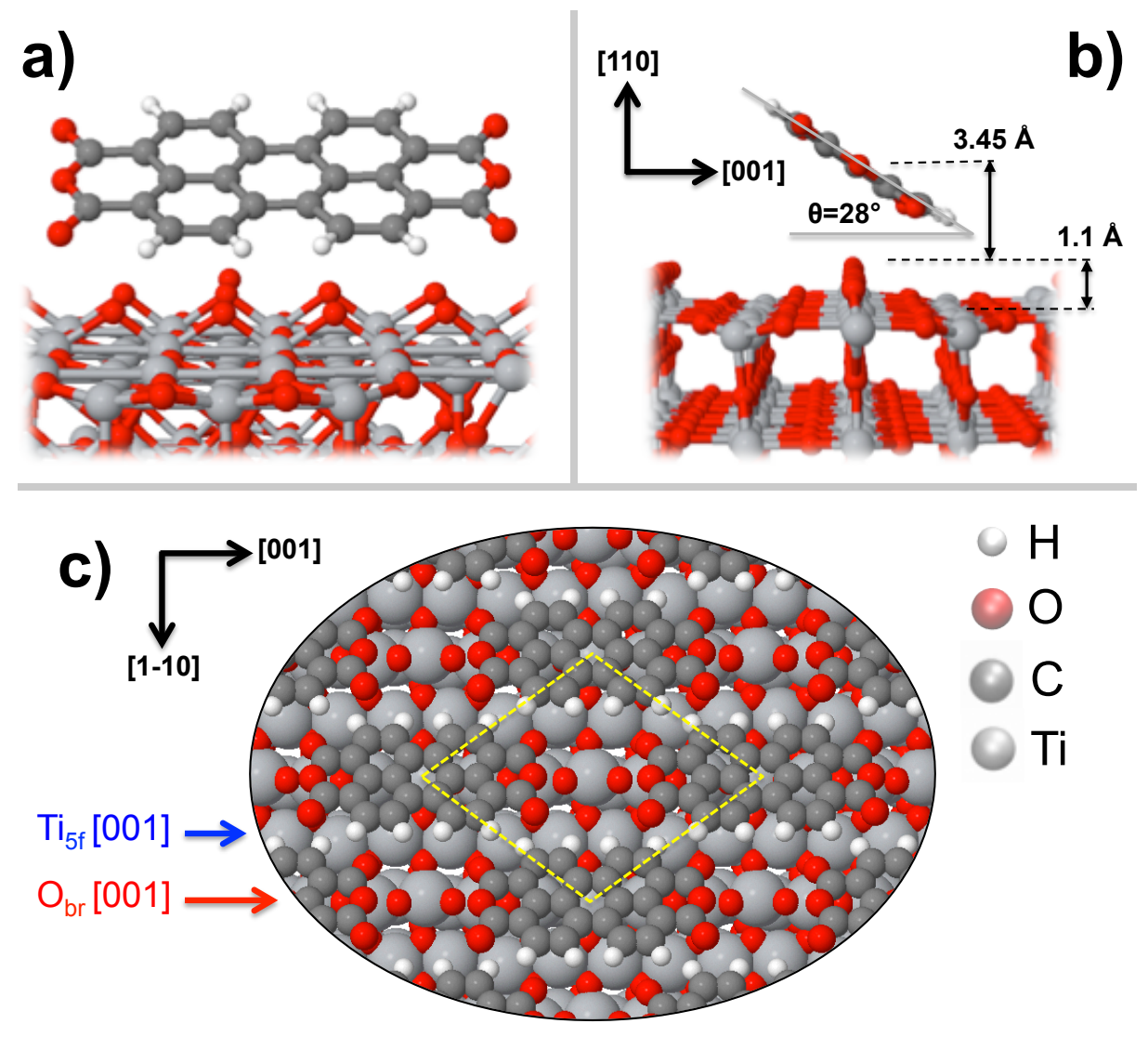

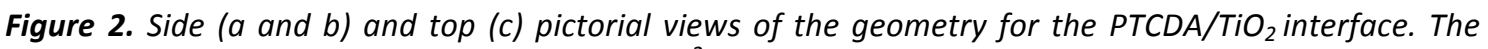
yellow-dashed rhomboid (c) of size $(11.2 \times 11.2) \AA^{2}$ and small angle $y=72.5^{\circ}$ shows the unit cell used in the calculations. $\mathrm{Ti}_{5 f}$ and $\mathrm{O}_{\mathrm{br}}$ high-symmetry rows of the oxide are also indicated.

\section{Experiment}

a)

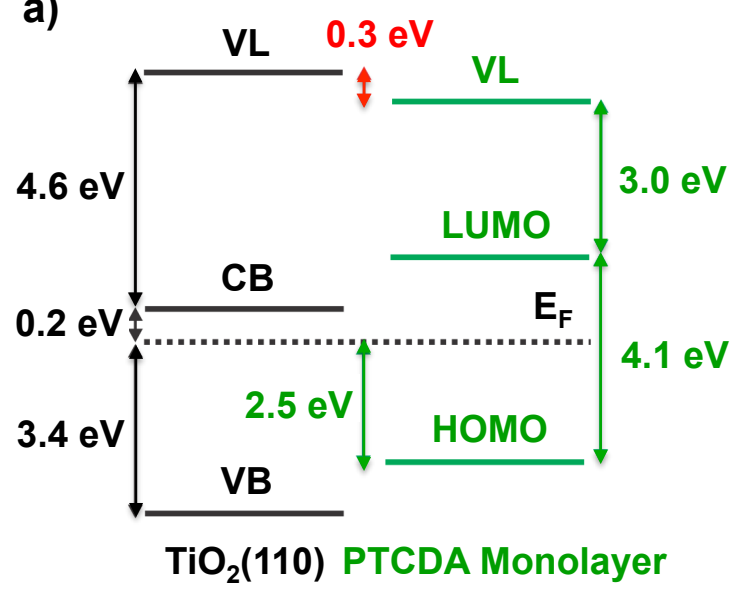

\section{Theory}

b)

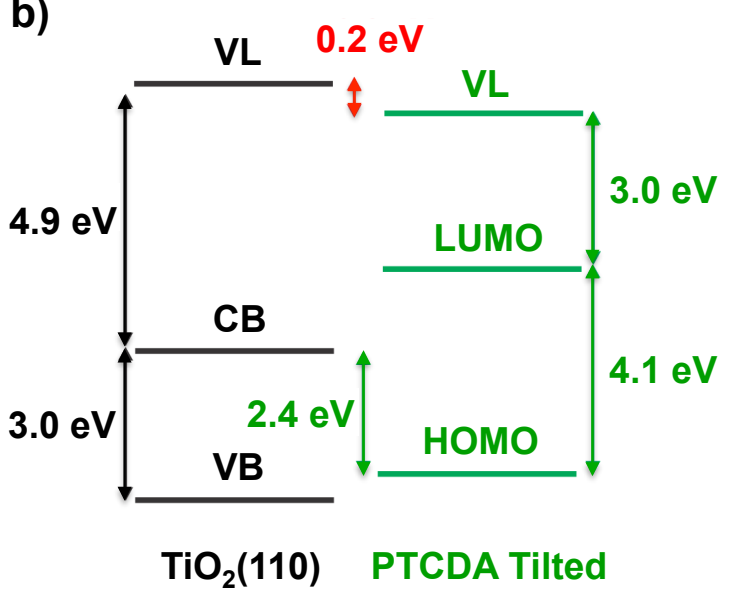

Figure 3. a) Experimental energy diagrams for a $1 \mathrm{ML}$ PTCDA adsorbed on $\mathrm{TiO}_{2}(110)$, compared to the b) theoretical energy alignment calculated for the tilted geometry (Figure 2).

\section{$\mathrm{Zn}-\mathrm{TPP} / \mathrm{TiO}_{2}(110)$ interface (chemisorbed regime)}

The overlayer geometry deduced from our experimental data and our DFT-calculations 
is shown in Figure $4 .{ }^{15}$ In this geometry the central $\mathrm{Zn}$ atom is placed $3.0 \AA$ directly above an oxygen atom of the oxide-O row. In this model geometry the Zn-TPP molecules form a monolayer with one molecule per unit cell with dimensions $16.4 \AA x$ $14.5 \AA$ (see Figure 4a). The Zn-TPP molecules become distorted with the Zn-TPP porphyrin ring adsorbed at a distance of 2.5-3.0 $\AA$ above the $\mathrm{TiO}_{2}(110)$ O-row; the mesophenyl rings of the molecule rotate by an angle between $2.5^{\circ}$ and $6.5^{\circ}$ with respect to the gas-phase geometry, so that those rings form an angle of $50 \pm 2^{\circ}$ with the flat porphyrin molecular skeleton, instead of $54.5^{\circ}$ in the gas phase; these rotations enable some of the $\mathrm{H}$ atoms of the mesophenyl rings to form weak bonds with the $\mathrm{O}$ atoms of the surface, such that the $\mathrm{H}-\mathrm{O}$ distances are between 2.2 and $2.4 \AA$.

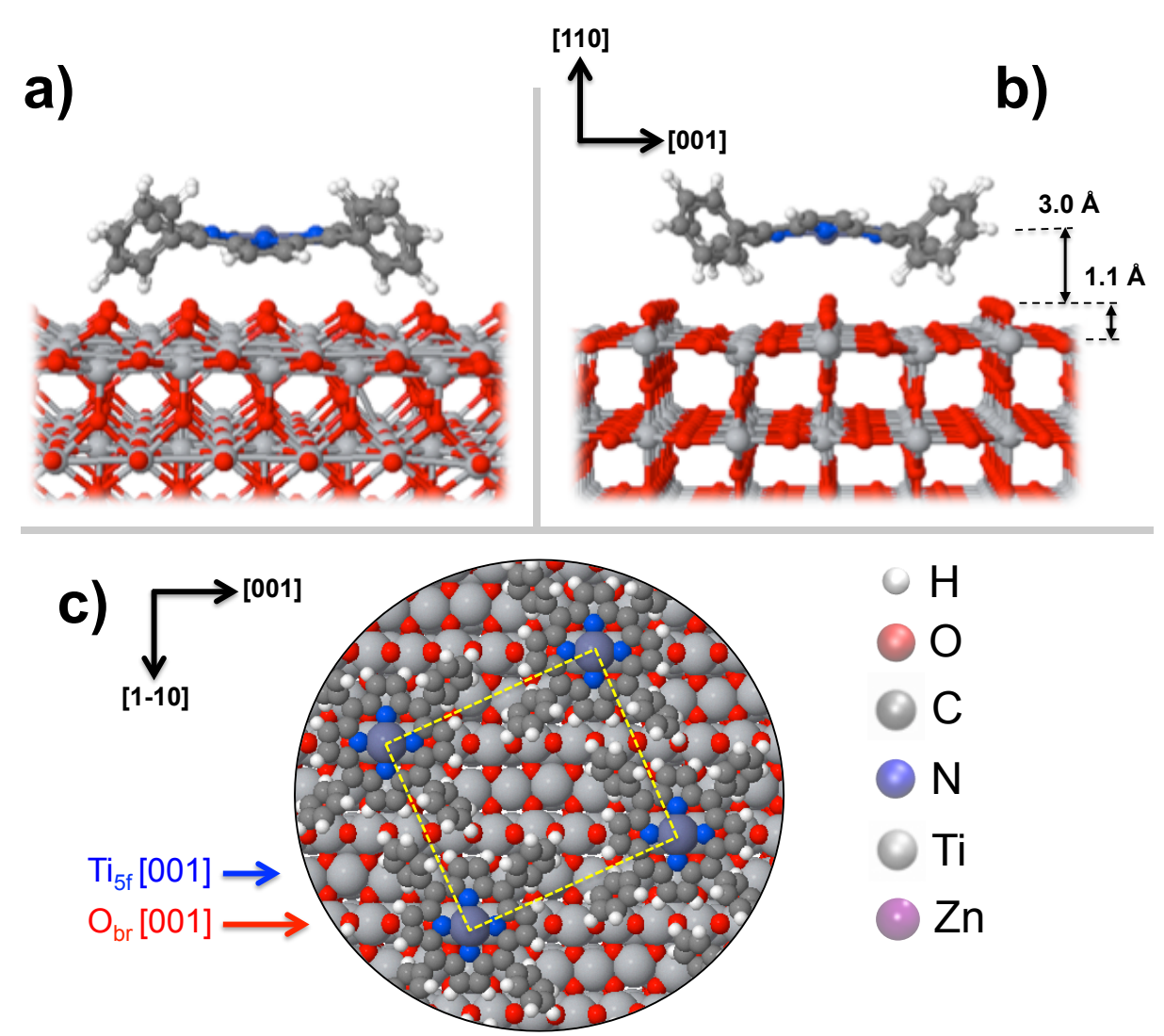

Figure 4. Side ( $a$ and b) and top (c) pictorial views of the geometry for the $\mathrm{Zn}$-TPP/TiO ${ }_{2}$ interface. The yellow-dashed rectangle of size $(16.4 \times 14.5) \AA^{2}$ shows the unit cell used in the calculations. $T i_{5 f}$ and $O_{b r}$ high-symmetry rows of the oxide are also indicated.

Figure 5 a shows the energy level diagram for the monolayer $\mathrm{Zn}-\mathrm{TPP} / \mathrm{TiO}_{2}(110)$ system that is drawn from the experimental measurements. ${ }^{15}$ The shift in the VLS of the clean and $\mathrm{Zn}$-TPP-covered $\mathrm{TiO}_{2}(110)$ surfaces indicates that there is an interface dipole potential of $0.9 \mathrm{eV}$. Figure $5 \mathrm{~b}$ shows the energy diagram as calculated with our theoretical approach ${ }^{15}$ for the monolayer geometry shown in figure 4 . The interface dipole potential is $0.85 \mathrm{eV}$, in good agreement with the experimental result; this dipole is mostly due to the electron charge transfer from the molecule to the oxide, which we find to be around 0.28 electrons per molecule suggesting a chemisorbed interaction between the organic layer and the oxide. As shown in figure 5, the organic HOMO level is inside the oxide gap while the LUMO level is located above the oxide conduction 
band edge.

\section{Experiment}

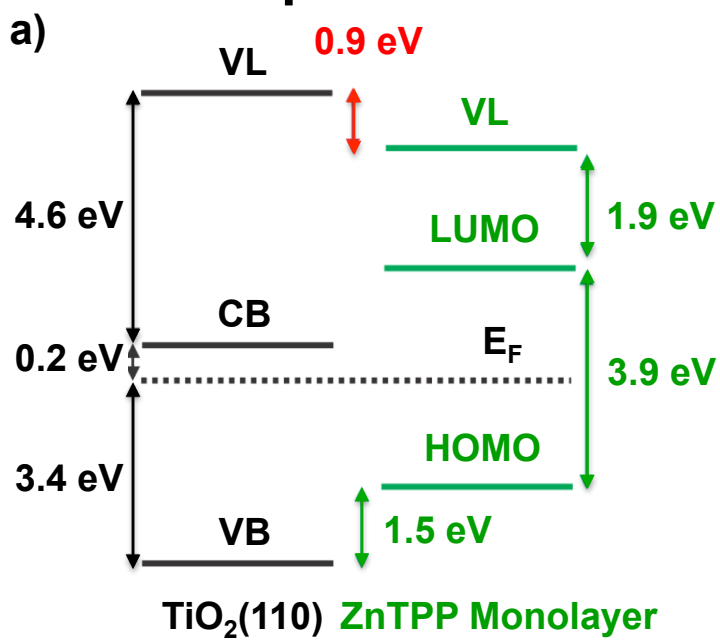

\section{Theory}

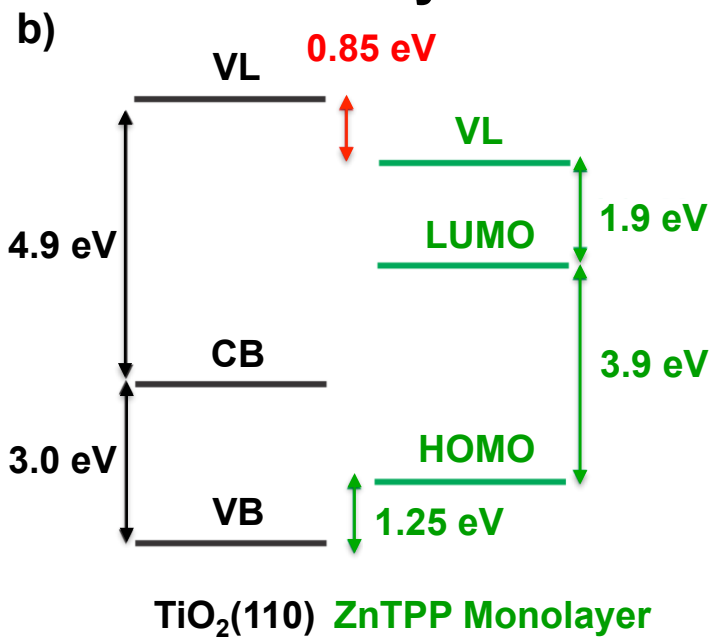

Figure 5. Energy level alignment for the $\mathrm{Zn}-\mathrm{TPP} / \mathrm{TiO}_{2}(110)$ interface: (a) Experimental results; (b) Theoretical results.

\section{$\mathrm{TCNQ} / \mathrm{TiO}_{2}(110)$ interface (strongly chemisorbed regime)}

Figure 6 shows the geometry calculated for the $\mathrm{TCNQ}_{\mathrm{TiO}}(110)$ interface: ${ }^{14}$ notice the important molecule bending induced by the strong chemical interaction between the molecule and the oxide. Our calculations ${ }^{14}$ show that the molecule forms strong bonds with the oxide; with its $\mathrm{N}$ atoms strongly bonded to the oxide $\mathrm{O}$ and $\mathrm{Ti}$ atoms. This adsorption geometry for the TCNQ molecule is similar to the one found for $\mathrm{TCNQ} / \mathrm{Au}(111) .{ }^{19}$

Figure 7 shows our calculations for the $\mathrm{TCNQ} / \mathrm{TiO}_{2}(110)$ interface at $0 \mathrm{~K}$ and room temperature. ${ }^{14}$ This case shows a peculiar difference with previous interfaces: the LUMO level is located below the oxide Fermi level and, consequently, an important charge transfer appears at room temperature from the oxide to that $L U M O$ level which is occupied by one electron. ${ }^{13,14}$ We stress that the oxide is $n$-doped, with the Fermi level located around $0.1 \mathrm{eV}$ from the conduction band. Due to that charge transfer a space charge layer $(S C L)$ appears in the oxide. ${ }^{13}$ Thus, we have analysed this problem in two steps: (a) first, we consider $\mathrm{T}=0 \mathrm{~K}$ when the electron charges are frozen in the oxide and, (b) we go to the room temperature case, when the electrons excited in the $\mathrm{n}$-doped oxide to the conduction band can be transferred to the organic molecules. 


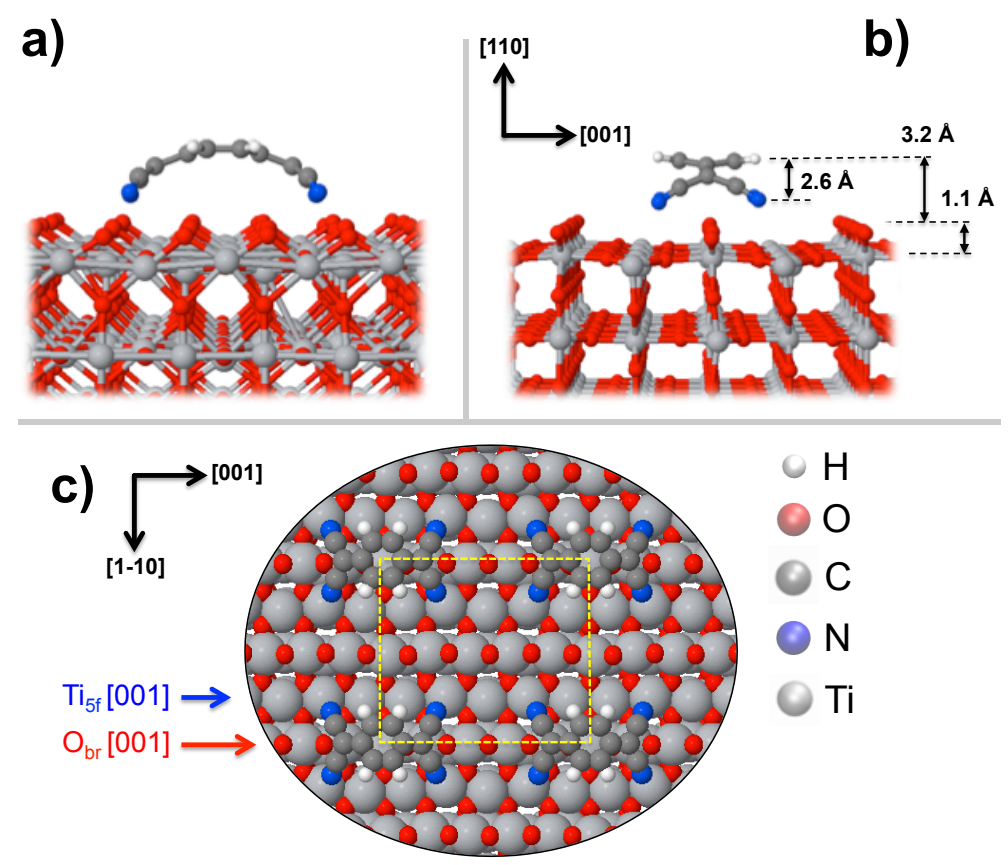

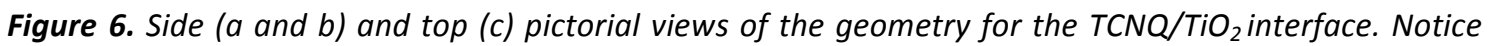
the pronounced on-surface molecule bending of $2.6 \AA$, which is the height of the molecule. The yellowdashed rectangle of size $(13.2 \times 15.0) \AA^{2}$ shows the unit cell used in the calculations. $T_{5 f}$ and $O_{b r}$ highsymmetry rows of the oxide are also indicated.

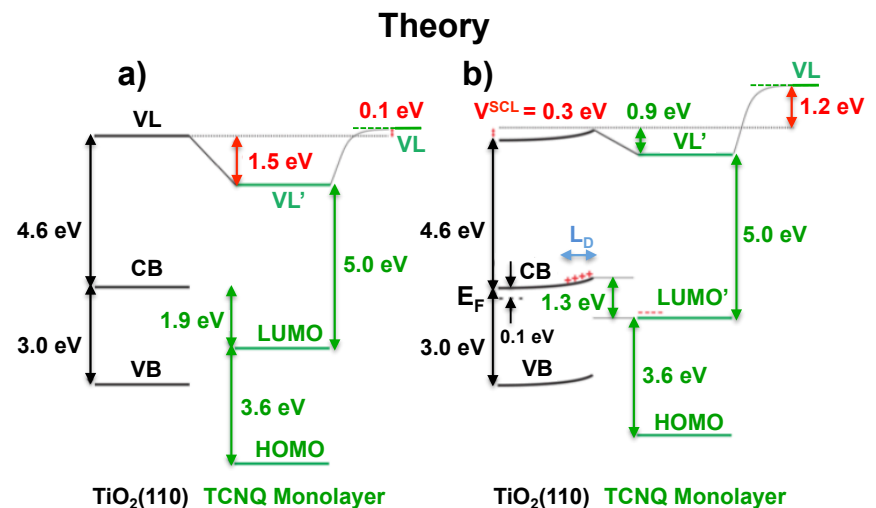

$\mathrm{TiO}_{2}(110) \mathrm{TCNQ}$ Monolayer

$\mathrm{TiO}_{2}(110) \mathrm{TCNQ}$ Monolayer

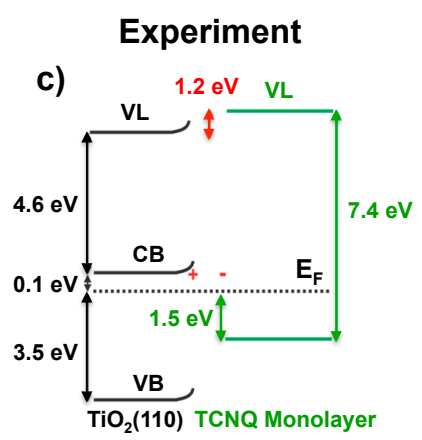

Figure 7. Energy level alignment calculated for the $T C N Q / T i O_{2}(110)$ interface at (a) $T=0 \mathrm{~K}$ and (b) room temperature. A $0.3 \mathrm{eV}$ band-bending is estimated at room temperature for $n$-doped $\mathrm{TiO}_{2}$. $\mathrm{VL}^{\prime}$ represents the initial vacuum level in the deformed gas-phase TCNQ-molecule with respect to the LUMO and HOMO levels (see also Figure $1 b$ ), in such a way that the difference between $V L$ and $V L^{\prime}$ is the hybridization shift introduced by the strong chemical interaction. (c) Energy diagram obtained from the experimental evidence. $^{14}$ 
Figure 7a shows the interface energy level alignment as calculated for $T=0 \mathrm{~K}$; at this temperature there is no charge transfer from the oxide $S C L$ to the $L U M O$ but we find an important interaction between the organic and the oxide surface atoms. Due to the corresponding strong oxide-molecule hybridization emerges a $1.6 \mathrm{eV}$ shift of the LUMO toward higher binding energies. ${ }^{14}$ At the same time, there is an electrostatic dipole of $0.1 \mathrm{eV}$ moving the LUMO level toward lower binding energies, in such a way that the LUMO is shifted by around $1.5 \mathrm{eV}$ toward higher binding energies, while the oxide work-function is increased by $0.1 \mathrm{eV}$ (see Figure 7a).

At room temperature, electrons are transferred from the conduction band of the $\mathrm{n}$ doped oxide to the $L U M O$, which becomes occupied by one electron. This populated LUMO is referred to as LUMO' because it is not formally, in this case, an unoccupied state. This transfer of charge creates an important electrostatic potential at the interface and a renormalization of the oxide-molecule hybridization shift that is increased to $2.1 \mathrm{eV}$. The new electrostatic interface dipole is due to (a) the space charge layer in the oxide and (b) to the potential created by the negative charge in the LUMO' and the corresponding opposite positive charge in the oxide. The space charge layer creates a surface potential of $0.3 \mathrm{eV}$ with respect to the oxide bulk, while the negative charge in the LUMO' (and its counter-positive charge in the oxide) creates an extra interface dipole of $1.1 \mathrm{eV}$ that shifts the organic levels to lower binding energies. The resulting interface energy diagram is shown in Figure $7 \mathrm{~b}$, which indicates an increase of $1.2 \mathrm{eV}$ of the oxide work-function, increasing by $1.1 \mathrm{eV}$ the value found for $\mathrm{T}=0 \mathrm{~K}$ due to the electronic filling of the $L U M O$ level.

The experimental evidence shows an upward band bending of $0.2 \mathrm{eV}$, which is a strong indication of an important charge transfer between the oxide and the organic monolayer. Moreover, this is accompanied by a VL-shift of $1.2 \mathrm{eV}$ (see figure 7c) also in good agreement with our theoretical analysis.

\section{Universal Trends in Organic/ $\mathrm{TiO}_{2}$ Interfaces}

Our results for PTCDA, Zn-TTP and TCNQ on TiO2 already show an interesting behaviour that depends on the organic electronegativity ${ }^{12}$ and the oxide/organic interaction. ${ }^{3,17}$ We can summarize those results in the following way: (a) First, TCNQ is the most electronegative with its LUMO level below the oxide conduction band edge $\left(E_{C}\right)$; at room temperature one electron is transferred from the oxide conduction band to the LUMO level, a space charge layer develops in the oxide surface and, consequently, the oxide work-function increases, $\approx 1.2 \mathrm{eV} .{ }^{13,14}$ (b) Zn-TTP shows a more conventional behaviour, with a chemisorbed interaction between the oxide and the organic. Then, some electron charge flows from the organic to the oxide and, consequently, there appears an important induced interface dipole and a decrease in the oxide work-function, $\approx-0.9 \mathrm{eV}^{3,15}$ (c) Finally, PTCDA shows a different behaviour, with a physisorbed regime due to the tilted geometry the molecule has when adsorbed on the oxide. In this regime the charge transfer from the molecule to the oxide is small and, consequently, there appears a very small decrease in the oxide work-function $\approx-0.3 \mathrm{eV} .^{12,17}$

We can understand better those results and, at the same time, to find a connection between all the cases discussed above if we analyse each oxide/organic interface 
behaviour as a function of a "virtual" potential, $\Delta$, which we apply "theoretically" between the organic and the oxide. $\Delta$ is an initial shift of all the energy levels of the organic molecule with respect to the oxide level before self-consistency. This shift, which mimics a change in the relative electronegativity of both materials, might be the result of applying an external potential between the two materials. In this calculation, we assume that the geometry of the interface does not change with $\Delta$.

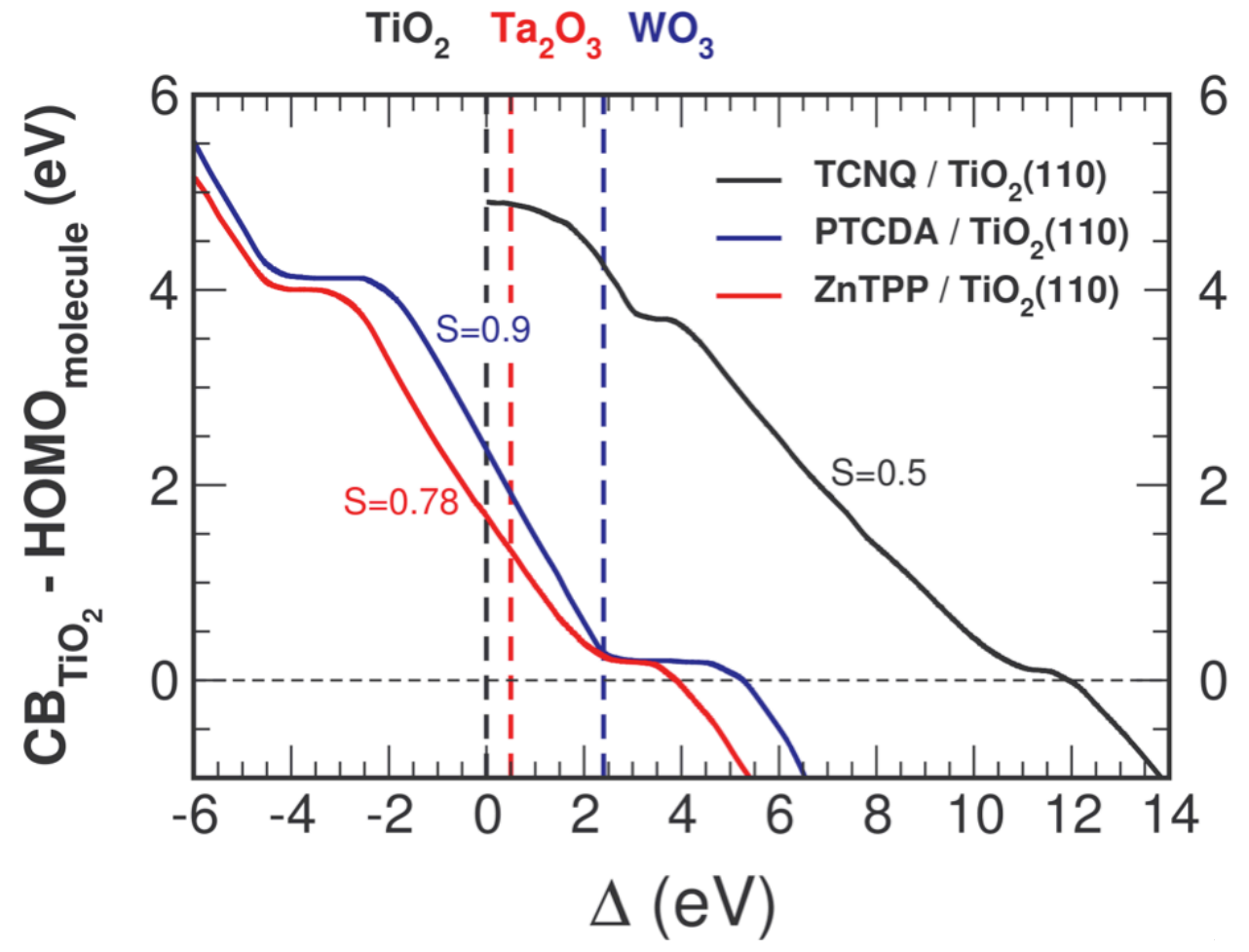

Figure 8. The oxide conduction band edge $\left(C B_{T i O 2}\right)$ minus the molecule HOMO level at RT conditions is depicted as a function of the relative positioning of the organic molecule and the oxide energy levels for PTCDA, Zn-TPP and TCNQ on $\mathrm{TiO}_{2}(110)$. This position is described via the $\triangle$ parameter, which defines by how much the organic levels are displaced with respect to the initial molecule levels. Visual guides: red and blue vertical dashed-lines have also been included in the figure to indicate how the interface properties for $\mathrm{Ta}_{2} \mathrm{O}_{3}$ and $\mathrm{WO}_{3}$, having similar gaps than $\mathrm{TiO}_{2}$, can be expected to depend on the different oxide affinity. $^{12}$

Figure 8 shows our theoretical results for the three oxide/organic interfaces, $\mathrm{PTCDA} / \mathrm{TiO}_{2}, \mathrm{Zn}-\mathrm{TPP} / \mathrm{TiO}_{2}$ and $\mathrm{TCNQ} / \mathrm{TiO}_{2}$ we are considering in this paper. In this figure we represent the value of $\left(E_{C}-H O M O_{\text {molecule }}\right)$ as a function of $\Delta$. Obviously, for $\Delta=0$ the values of $\left(E_{C}-H O M O_{\text {molecule }}\right)$ correspond to the ones shown in Figures $3 \mathrm{~b}, 5 \mathrm{~b}$ and $7 \mathrm{~b}$. In Figure 8 we have drawn two dashed vertical lines corresponding to the different electron affinities of $\mathrm{WO}_{3}$ and $\mathrm{Ta}_{2} \mathrm{O}_{3}$, oxides that have similar energy gaps; taking these lines as the origin for each case, we can expect to simulate the interface properties for these oxides assuming similar chemical interactions as $\mathrm{TiO}_{2}$. It should be stressed that in these calculations, and for the $\Delta$-window for which a SCL appears, we have assumed that the oxide Fermi level is $0.1 \mathrm{eV}$ below the conduction band edge; small changes in this value will modify the Debye-length, $L_{D}\left\{L_{D}=V\left(\varepsilon k_{B} T / 4 \pi e n_{e}\right),{ }^{14} n_{e}\right.$ being the electron charge density of the doped material\} and the SCL-potential, $V^{S C L}$ (see figure $7 b$ ). Regarding the values presented in figure 8 , a change in the oxide Fermi 
level would only change the $\left(C B_{T i O 2}-H O M O_{\text {molecule }}\right)$-values by a constant, namely, the shift in the position of the oxide Fermi level.

Figure 8 results summarize and extend our previous calculations and show the differences and similarities between the different molecules. In particular, we find that:

- All the cases show a similar qualitative behavior with a displacement between different curves due to the different organic electronegativity. For example, the $\mathrm{TCNQ} / \mathrm{TiO}_{2}$ curve, for the most electronegative organic, exhibits the largest displacement towards positive values of $\Delta$.

- The slope of the central part of those curves is $(-S)$ with $S=d /\left(E_{C}-H O M O\right) / d \Delta$, which we find to be: $S \approx 0.90$ for PTCDA/TiO $2(110) ; S \approx 0.78$ for $\mathrm{Zn}-\mathrm{TPP} / \mathrm{TiO}_{2}(110)$; and $S \approx 0.5$ for $\mathrm{TCNO} / \mathrm{TiO}_{2}(110)$, with $S$ depending on the organic/oxide interaction, and close to 1 for a physisorbed oxide/organic interaction.

- We also find in all the curves two flat regions, with ( $\left.E_{C}-H O M O\right)$ constant as a function of $\Delta$. That behavior corresponds to having the LUMO or the HOMO levels crossing the oxide Fermi level; along that flat behavior, electron charge is transferred from the oxide-SCL to the $\angle U M O$, or from the HOMO to that oxide$S C L$, in such a way that the organic levels remain constant until one electron is transferred to the $L U M O$ (or from the HOMO) level. This finding evidences a prototypical Coulomb blockade regime, with the LUMO or the HOMO levels being pinned by the oxide Fermi level. It is interesting to stress that, along that Coulomb blockade regime a space charge layer develops in the oxide surface, being this fact a clear signal of having that regime; we should stress that, as already mentioned above, the space charge layer potential would depend on the oxide $n$-doping which in our experiments and theory is characterized by an oxide Fermi level located $0.1 \mathrm{eV}$ from the conduction band edge. On the other hand, it is important to notice that the Coulomb blockade regime extends for an interval value of $\Delta$ that is directly related to the effective coulomb interaction, $U^{\text {eff }}$, associated with the organic monolayer. ${ }^{14}$ In this regime one electron is transferred to (from) the LUMO (HOMO) level, that charge creating an electrostatic potential, $U^{\text {eff }}$, that opposes that charge transfer; however, due to the interface screening that electrostatic potential is reduced to $S U^{\text {eff }}$. Notice that $U^{\text {eff }}$, slightly larger than $U_{1}^{14}$ incorporates also the electrostatic potential created by all the charges associated with the monolayer, while $U$ in equation (1) is mainly a single molecule effect that takes into account polarization effects due mainly to the image potential.

\section{Summary and Conclusions}

In conclusion, Figure 8 characterizes the universal behavior of the oxide/organic interfaces. In the most conventional limit, the HOMO level is located in the oxide energy gap below the oxide Fermi level, and the LUMO level above; in this regime, depending on the interaction between the oxide and the organic, some electronic charge is transferred from the organic to the oxide, resulting in a decrease of the oxide 
work-function. That interaction can be characterized by the following parameter: $S=$ $d /\left(E_{C}-H O M O\right) / d \Delta$ which for a physisorbed interaction is close to 1 ; however, for a chemisorbed regime, $S$ might be in the range 0.5-0.9.

At the edges of this central part of the $\left\{\left(E_{C}-H O M O\right)\right.$ vs $\left.\Delta\right\}$ curve, we find in all the cases two plateaus, which represent two Coulomb blockade regimes, for which the LUMO or the HOMO levels are pinned by the oxide Fermi level. At the same time that this regime appears, the oxide develops a space surface layer, which accommodates the extra charge (negative or positive) that has been transferred to the organic.

Finally, once one electron has been transferred from (to) the oxide to (from) the organic, the system evolves with the organic levels being displaced as a function of $\Delta$ in a similar way to the behavior found in the central part of the $\left\{\left(E_{C}-H O M O\right)\right.$ vs $\left.\Delta\right\}$ curve. Those regimes correspond to having the LUMO (HOMO) level below (above) the oxide Fermi energy level.

Our analysis shows that the universal behavior of the oxide/organic interface alignment is characterized basically by a zig-zag curve for the $\left\{\left(E_{C}-H O M O\right)\right.$ vs $\left.\Delta\right\}$ representation, with a slope between 0.5 and 1 in the central and lateral parts, depending on the oxide/organic interaction, and two plateaus representing Coulomb blockade regimes associated with the alignment between the LUMO or HOMO levels with the oxide Fermi energy. Different organic materials show that kind of universal behavior but displaced in the $\Delta$-scale towards positive values of $\Delta$ for more electronegative organic molecules.

\section{Acknowledgements}

SR, CR and RAB acknowledge support from the Department of Energy under Grant No. FG02-01ER15256. CR acknowledges support from the Peter Lindenfeld Graduate Fellowship. FF and JO acknowledge support from the Spanish Ministerio de Economía y Competitividad (MINECO) under Grant No. MAT2014-59966-R and through the "María de Maeztu" Program for Units of Excellence in R\&D (Grant No. MDM-2014-0377). JIM acknowledges financial support by the Spanish MINECO via the "Ramón y Cajal" Program (Grant No. RYC-2015-17730), and the EU via the ERC-Synergy Program (Grant No. ERC-2013-SYG-610256 NANOCOSMOS) and the Innovation Program under grant agreement No. 696656 (GrapheneCore1-Graphene-based disruptive technologies).

\section{Notes and references}

$1 \quad$ S. R. Forrest, Chem Rev 1997, 97, 1793-1896.

2 D. Kabra, L. P. Lu, M. H. Song, H. J. Snaith, R. H. Friend, Adv. Mater. 2010, 22, 3194-3198.

3 H. Li, P. Winget, P., J.-L. Brédas, Chem. Mat. 2014, 26, 631-646.

4 J. Meyer, S. Hamwi, M. Kroger, W. Kowalsky T. Riedl, A. Kahn, Adv. Mater. 2012, 24, 5408-5427.

$5 \quad$ M. Sessolo, H. J. Bolink, Adv. Mater. 2011, 23, 1829-1845.

6 D. Cahen, A. Kahn, E. Umbach, Mater Today 2005, 8, 32-41.

7 F. Flores, J. Ortega, H. Vazquez, Phys. Chem. Chem. Phys. 2009, 11, 8658-8675.

$8 \quad$ N. Koch, J. Phys.: Condens. Matter 2008, 20, 184008.

9. M. Fahlman, A. Crispin, X. Crispin, K. M. Henze, M. P. de Jong, W. Osikowicz, C. 
Tengstedt, W. R. Salaneck, J. Phys.: Condens. Matter 2007, 19, 183202.

10 D. Beljonne, J. Cornil, L. Muccioli, C. Zannoni, J.-L. Brédas, F. Castet, Chem. Mater. 2011, 23, 591-609.

11 P. Bagus, V. Staemmler, C. Wöll, Phys. Rev. Lett. 2002, 89, 96104; H. Vázquez, Y. J. Dappe, J. Ortega, F. Flores, J. Chem. Phys. 2007, 126, 144703.

12 M. T. Greiner, M. G. Helander, W.-M. Tang, Z.-B. Wang, J. Qiu, Z.-H. Lu, Nat. Mater. 2012, 11, 76-81.

13 Y. Xu, Phys. Rev. Lett. 2013, 111, 226802.

14 J. I. Martínez, F. Flores, J. Ortega, S. Rangan, C. Ruggieri, R. A. Bartynski, J. Phys. Chem. C 2015, 119, 22086-22091.

15 S. Rangan, C. Ruggieri, R. A. Bartynski, J. I. Martínez, F. Flores, J. Ortega, J. Phys. Chem. C 2016, 120, 4430-4437.

16 P. Winget, et al., Adv. Mater. 2014, 26, 4711-4716.

17 W. Chen, C. Tegenkamp, H. Pfnur, T. Bredow, J. Chem. Phys. 2010, 132, 214706-

5.

18 S. Rangan, C. Ruggieri, R. A. Bartynski, J. I. Martínez, F. Flores, J. Ortega, J. Phys. Chem. B 2017 (in press; DOI: 10.1021/acs.jpcb.7b04227).

19 J. I. Martínez, E. Abad, F. Flores, J. Ortega, Phys. Stat. Sol. B 2011, 248, 20442049.

20 P. Giannozzi, et al., J. Phys.: Condens. Mat. 2009, 21, 395502.

21 P. Jelínek, H. Wang, J. P. Lewis, O. F. Sankey, J. Ortega, Phys. Rev. B 2005, 71, 235101.

22 J. P. Lewis, K. R. Glaesemann, G. A. Voth, J. Fritsch, A. A. Demkov, J. Ortega, O. F. Sankey, Phys. Rev. B 2001, 64, 1989.

23 J. P. Lewis, P. Jelínek, J. Ortega, A. A. Demkov, D. G. Trabada, B. Haycock, H. Wang, G. Adams, J. K. Tomfohr, E. Abad, H. Wang, D. A. Drabold, Phys. Stat. Sol. B 2011, 248, 1989-2007.

24 J. P. Perdew, K. Burke, M. Ernzerhof, Phys. Rev. Lett. 1996, 77, 3865-3868.

25 A. M. Rappe, K. M. Rabe, E. Kaxiras, E., J. D. Joannopoulos, Phys. Rev. B 1990, 41, 1227.

26 N. Mounet, N. Marzari, Phys. Rev. B 2005, 71, 205214.

27 H. J. Monkhorst, J. D. Pack, Phys. Rev. B 1976, 13, 5188-5192.

28 V. Barone, M. Casarin, D. Forrer, M. Pavone, M. Sambi, A. Vittadini, J. Comput. Chem. 2009, 30, 934-939.

29 S. Grimme, J. Comput. Chem. 2006, 27, 1787-1799.

30 M. Methfessel, A. T. Paxton, Phys. Rev. B 1989, 40, 3616-3621.

31 M. A. Basanta, Y. J. Dappe, P. Jelínek, J. Ortega, Comp. Mater. Sci. 2007, 39, 759-766.

32 J. I. Martínez, E. Abad, C. González, F. Flores, J. Ortega, Phys. Rev. Lett. 2012, $108,246102$.

33 M. Fuchs, M. Scheffler, Comput. Phys. Commun. 1999, 119, 67-98.

34 Gaussian 09, Revision E.01, M. J. Frisch, et al., Gaussian, Inc., Wallingford CT (2009).

35 J. I. Beltrán, F. Flores, J. I. Martínez, J. Ortega, J. Phys. Chem. C 2013, 117, 38883894.

36 J. I. Martínez, E. Abad, J. I. Beltrán, F. Flores, J. Ortega, J. Chem. Phys. 2013, 139, 214706. 
37 A. Biller, I. Tamblyn, J. B. Neaton, L. Kronik, J. Chem. Phys. 2011, 135, 164706.

38 W. Chen, C. Tegenkamp, H. Pfnur, T. Bredow, J. Chem. Phys. 2010, 132, 214706.

39 C. Freysoldt, P. Rinke, M. Scheffler, Phys. Rev. Lett. 2009, 103, 056803.

40 L. Kronik, R. Fromherz, E. Ko, G. Gantefor, J. R. Chelikowsky, Eur. Phys. J. D 2003, 24, 33-36.

41 E. Abad, Y. J. Dappe, J. I. Martínez, F. Flores, J. Ortega, J. Chem. Phys. 2011, 134, 044701.

42 S. Godlewski, J. S. Prauzner-Bechcicki, T. Glatzel, E. Meyer, M. Szymonski, Beilstein J. Nanotech. 2015, 6, 1498-1507.

43 S. Godlewski, A. Tekiel, W. Piskorz, F. Zasada, J. S. Prauzner-Bechcicki, Z. Sojka, M. Szymonski, ACS Nano 2012, 6, 8536-8545.

44 V. Lanzilotto, G. Lovat, G. Otero, L. Sánchez, M. F. López, J. Méndez, J. A. Martín-Gago, G. Bavdek, L. Floreano, J. Phys. Chem. C 2013, 117, 12639-12647.

45 G. Otero-Irurueta, J. I. Martínez, G. Lovat, V. Lanzilotto, J. Méndez, M. F. López, L. Floreano, J. A. Martín-Gago, J. Phys. Chem. C 2015, 119, 7809-7816.

46 V. Lanzilotto, C. Sanchez-Sanchez, G. Bavdek, D. Cvetko, M. F. López, J. A. Martín-Gago, L. Floreano, J. Phys. Chem. C 2011, 115, 4664-4672. 

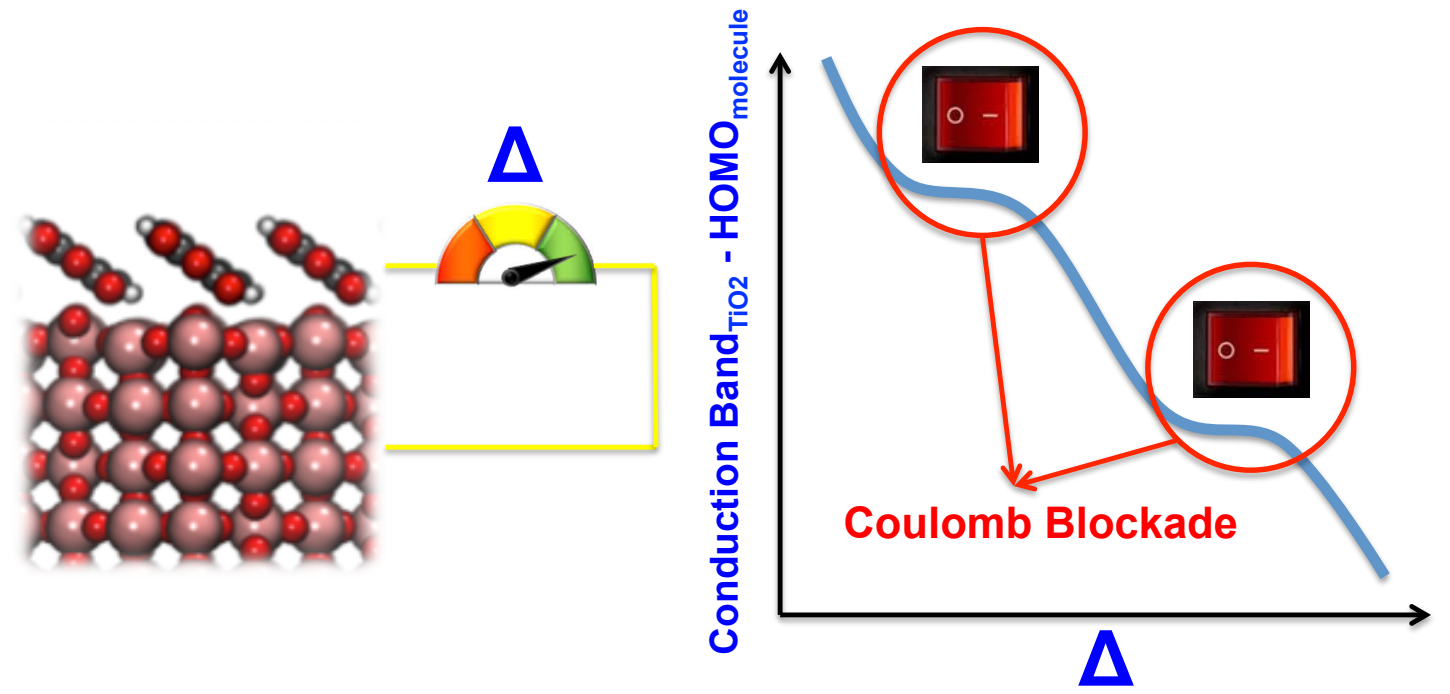

TOC. Organic/oxide interfaces exhibit an energy-level-alignment universal behaviour when a bias is applied. Coulomb-blockade regime is ruled by the organic electronegativity. 